\title{
Some New Dual hesitant fuzzy power aggregation operator
}

\author{
Jiayong Xing ${ }^{1, a}$, Rui $\mathrm{Yu}^{2, \mathrm{~b}, *}$ and Guangchun Zhang ${ }^{1, c}$ \\ ${ }^{1}$ College of Communications Engineering, PLA University of Science and Technology, Nanjing, \\ 210000, China \\ ${ }^{2}$ Institute of China Electronic System Engineering Corporation, Beijing, 100000, China \\ ajiayongxingnj@yeah.net, byuruinj@yeah.net, zgcnj@126.com
}

Keywords: Dual hesitant fuzzy, power aggregation operator, multiple attribute group decision making.

\begin{abstract}
Multi-criteria decision making (MCDM) has been a hot topic in decision making and systems engineering. The Dual Hesitant Fuzzy Sets (DHFSs) is a useful tool to deal with vagueness and ambiguity in the MADM problems. In this paper, we propose some new dual hesitant fuzzy power aggregation operator for dual hesitant fuzzy information. We first redefine some basic operations of dual hesitant fuzzy sets, which are consistent with those of dual hesitant fuzzy sets. Then we propose several power aggregation operator on dual hesitant fuzzy sets, study their properties and give some specific dual hesitant fuzzy aggregation operator. In the end, we develop one approache for multiple attribute group decision making with dual hesitant fuzzy information, and illustrate a real world example to show the behavior of the proposed operator.
\end{abstract}

\section{Introduction}

Zhu and $\mathrm{Xu}$ introduced the definition of dual hesitant fuzzy set ${ }^{[1]}$, which used the membership hesitancy function and the non-membership hesitancy function to support a more exemplary and flexible access to assign values for each element in the domain. DHFS can be regarded as a more comprehensive set, which supports a more flexible approach when the decision makers provide their judgments. The existing sets, including fuzzy sets (FSs) ${ }^{[2]}$, intuitionistic fuzzy sets (IFSs) ${ }^{[3,4]}$ and hesitant fuzzy sets (HFSs) ${ }^{[5,6]}$ can be regarded as special cases of DHFSs.

The objective in multiple attribute group decision making (MAGDM) problems is to find the most desirable alternative(s) among a set of feasible alternatives, based on the preferences provided by a group of experts. The fundamental prerequisite of decision making is how to aggregate individual experts' preference information on alternatives. Information aggregation is a process that combines individual experts' preferences into an overall one by using a proper aggregation technique ${ }^{[7]}$. Aggregation operator are the most widely used tool for combining individual preference information into overall preference information and deriving collective preference values for each alternative. The investigation on information aggregation has received surprisingly extensive attention from practitioners and researchers due to its practical and academic significance. Yager ${ }^{[8]}$ developed a power average (PA) operator and a power ordered weighted average (POWA) operator to provide aggregation tools for which the weight vectors depend on the input arguments and that allow the values being aggregated to support and reinforce one another. Motivated by Yager, Xu and Yager ${ }^{[9]}$ proposed a power geometric (PG) operator and a power ordered weighted (POWG) operator. However, the arguments of these power aggregation operators are exact numerical values. In practice, we often encounter situations in which the input arguments cannot be expressed as exact numerical values. Zhou et al. ${ }^{[10]}$ presented the generalized power average (GPA) operator and the generalized power ordered weighted average (GPOWA) operator. $\mathrm{Xu}^{[11]}$ and Zhou et al. ${ }^{[12]}$ extended the PA, POWA, PG, and POWG operators to intuitionistic fuzzy environments and developed some intuitionistic fuzzy power aggregation operators. Zhang ${ }^{[13]}$ developed a series of generalized intuitionistic fuzzy power geometric operators to aggregate input arguments that are intuitionistic 
fuzzy numbers and studied some desired properties of these aggregation operators and investigate the relationships among these operators.

In the real world, when experts make a decision, they are usually hesitant and irresolute for one thing or another which makes it difficult to reach a final agreement. They further indicated that DHFSs can better deal with the situations that permit both the membership and the no-membership of an element to a given set having a few different values, which can arise in a group decision making problem. It is, therefore, necessary to extend the existing power aggregation operator to dual hesitant fuzzy environments and to develop new power aggregation operator for aggregating dual hesitant fuzzy information. In this paper, we first review the dual hesitant fuzzy, and then give some other dual hesitant fuzzy operations. We further propose some dual hesitant fuzzy power aggregation operator.

To do this, the remainder of the paper is organized as follows. Section 2 presents some basic concepts related to DHFSs and PA operator. In Section 3, we present one new dual hesitant fuzzy power aggregation operator, investigate some of their basic properties. In Section 4, we propose a one approache based on DHFSs and then use a numerical example to illustrate our operator. Finally, Section 5 concludes the paper with some remarks and presents future challenges.

\section{Preliminaries}

\subsection{DHFSs.}

Definition 1 [1]. Let $X$ be a fixed set, then a dual hesitant fuzzy set (DHFS) $D$ on $X$ is described as:

$D=\{<x, h(x), g(x)>\mid x ? X\}$

In which $h(x)$ and $g(x)$ are two sets of some values in $[0,1]$, denoting the possible membership degrees and non-membership degrees of the element $x \hat{\mathrm{I}} X$ to the set $D$ respectively, with the conditions:

$0 \# g, h \quad 1,0 ? g^{+} \quad h^{+}$? 1

Where $g \hat{\mathrm{I}} h(x), h \hat{\mathrm{I}} g(x), g^{+} \hat{\mathrm{I}} h^{+}(x)=\mathrm{U}_{g \hat{\mathrm{I}} h(x)} \max \{g\}$, and $h^{+} \hat{\mathrm{I}} g^{+}(x)=\mathrm{U}_{h \hat{\mathrm{I}} g(x)} \max \{h\}$ for all $x$ I $X$. For convenience, the pair $a(x)=(h(x), g(x))$ is called a dual hesitant fuzzy element (DHFE), denoted by $a=(h, g)$, with the conditions: $g \hat{\mathrm{I}} h(x), h \hat{\mathrm{I}} g(x)$, $g^{+} \hat{\mathrm{I}} h^{+}(x)=\mathrm{U}_{g \hat{\mathrm{I} h(x)}} \max \{g\}$, and $h^{+} \hat{\mathrm{I}} g^{+}(x)=\mathrm{U}_{h \hat{\mathrm{I} g(x)}} \max \{h\}, 0 \# g, h \quad 1$ and $0 ? g^{+} \quad h^{+} ? 1$.

Definition 2 [1] Let $X$ be a fixed set, $a_{1}$ and $a_{2}$ two DHFEs, Then:

(1) $\alpha_{1} \mathrm{U} \alpha_{2}=\left\{h_{\alpha} \in\left(h_{\alpha_{1}} \mathrm{U} h_{\alpha_{2}}\right)\left|h_{\alpha} \geq \max \left(h_{\alpha_{1}}^{-}, h_{\alpha_{2}}^{-}\right), g_{\alpha} \in\left(g_{\alpha_{1}} \mathrm{I} g_{\alpha_{2}}\right)\right| g_{\alpha} \leq \min \left(g_{\alpha_{1}}^{+}, g_{\alpha_{2}}^{+}\right)\right\}$;

(2) $\alpha_{1} \mathrm{I} \quad \alpha_{2}=\left\{h_{\alpha} \in\left(h_{\alpha_{1}} \mathrm{I} h_{\alpha_{2}}\right)\left|h_{\alpha} \leq \min \left(h_{\alpha_{1}}^{+}, h_{\alpha_{2}}^{+}\right), g_{\alpha} \in\left(g_{\alpha_{1}} \mathrm{U} g_{\alpha_{2}}\right)\right| g_{\alpha} \geq \max \left(g_{\alpha_{1}}^{-}, g_{\alpha_{2}}^{-}\right)\right\}$

(3) $\alpha_{1} \oplus \alpha_{2}=\mathrm{U}_{\gamma_{\alpha_{1}} \in h_{\alpha_{1}}, \eta_{\alpha_{1}} \in g_{\alpha_{1}}, \gamma_{\alpha_{2}} \in h_{\alpha_{2}}, \eta_{\alpha_{2}} \in g_{\alpha_{2}}}\left\{\left\{\gamma_{\alpha_{1}}+\gamma_{\alpha_{2}}-\gamma_{\alpha_{1}} \gamma_{\alpha_{2}}\right\},\left\{\eta_{\alpha_{1}} \eta_{\alpha_{2}}\right\}\right\}$;

(4) $\alpha_{1} \otimes \alpha_{2}=\mathrm{U}_{\gamma_{\alpha_{1}} \in h_{\alpha_{1}}, \eta_{\alpha_{1}} \in g_{\alpha_{1}}, \gamma_{\alpha_{2}} \in h_{\alpha_{2}}, \eta_{\alpha_{2}} \in g_{\alpha_{2}}}\left\{\left\{\gamma_{\alpha_{1}} \gamma_{\alpha_{2}}\right\},\left\{\eta_{\alpha_{1}}+\eta_{\alpha_{2}}-\eta_{\alpha_{1}} \eta_{\alpha_{2}}\right\}\right\}$;

(5) $n \alpha=\mathrm{U}_{\gamma_{\alpha} \in h_{\alpha}, \eta_{\alpha} \in g_{\alpha}}\left\{1-\left(1-\gamma_{\alpha}\right)^{n},\left(\eta_{\alpha}\right)^{n}\right\}$;

(6) $\alpha^{n}=\mathrm{U}_{\gamma_{\alpha} \in h_{\alpha}, \eta_{\alpha} \in g_{\alpha}}\left\{\left(\gamma_{\alpha}\right)^{n}, 1-\left(1-\eta_{\alpha}\right)^{n}\right\}$.

where $n$ is a positive integral and all the results are also DHFEs.

To compare the DHFEs, Zhu and Xu gave the following comparison laws:

Definition 3 [1]. Let $a_{i}=\left\{h_{d_{i}}, g_{d_{i}}\right\}(i=1,2)$ be any two DHFEs, the score function of $a$ and the accuracy function of $a$, is defined as: 


$$
\begin{aligned}
& s(a)=\frac{1}{\# h} ?_{g \text { 谜 }} g \frac{1}{\# g} ?_{h g} h \\
& p(a)=\frac{1}{\# h} ?_{g \text { 谜 }} g \quad \frac{1}{\# g} ?_{h g} h
\end{aligned}
$$

where $\# h$ and $\# g$ are the numbers of the elements in $h$ and $g$ respectively, then

if $s_{\alpha_{1}}>s_{\alpha_{2}}$, then $\alpha_{1}$ is superior to $\alpha_{2}$, denoted by $\alpha_{1} \mathrm{f} \alpha_{2}$;

if $s_{\alpha_{1}}=s_{\alpha_{2}}$, then

(1) if $p_{\alpha_{1}}=p_{\alpha_{2}}$, then $\alpha_{1}$ is equivalent to $\alpha_{2}$, denoted by $\alpha_{1} \sim \alpha_{2}$;

(2) If $p_{\alpha_{1}}>p_{\alpha_{2}}$, then $\alpha_{1}$ is superior than $\alpha_{2}$, denoted by $\alpha_{1} \mathrm{f} \alpha_{2}$.

However, little has been done about distance and similarity measures of DHFEs. We first introduce the concept of distance of DHFEs and then propose some new distance measures for DHFSs.

\subsection{Power aggregation operator.}

Yager [81] introduced a nonlinear weighted-average aggregation tool, which is called power average operator as follows:

Definition $4\left[\right.$ ]. The power average (PA) operator is the mapping PA: $R^{n} \rightarrow R$ defined by the following formula:

$$
P A\left(a_{i} \mid i=1,2, \mathrm{~K}, n\right)=\frac{\sum_{i=1}^{n}\left(1+T\left(a_{i}\right)\right) a_{i}}{\sum_{i=1}^{n}\left(1+T\left(a_{i}\right)\right)},
$$

Where

$$
T\left(a_{i}\right)=\sum_{\substack{j=1 \\ j \neq i}}^{n} \operatorname{Sup}\left(a_{i}, a_{j}\right)
$$

And $\operatorname{Sup}\left(a_{i}, a_{j}\right)$ is the support for $a_{i}$ from $a_{j}$. The support satisfies the following three properties:

$$
\begin{aligned}
& \text { 1) } \operatorname{Sup}\left(a_{i}, a_{j}\right) \in[0,1] \text {; } \\
& \text { 2) } \operatorname{Sup}\left(a_{i}, a_{j}\right)=\operatorname{Sup}\left(a_{j}, a_{i}\right) \text {; } \\
& \text { 3) } \operatorname{Sup}\left(a_{i}, a_{j}\right) \geq \operatorname{Sup}\left(a_{s}, a_{t}\right) \text { if }\left|a_{i}-a_{j}\right|<\left|a_{s}-a_{t}\right| .
\end{aligned}
$$

\section{Dual hesitant fuzzy power average (DHFPA) operator}

Definition 11 Let $a_{i}=\left\{h_{a_{i}}, g_{a_{i}}\right\}(i=1,2, \mathrm{~K}, n)$ be a collection of DHFEs, a Dual hesitant fuzzy power average power average (DHFPA) operator and a Dual hesitant fuzzy power average power average (DHFPG) operator are defined by the following formula:

$$
\begin{aligned}
& \operatorname{DHFPA}\left(\alpha_{i} \mid i=1,2, \mathrm{~K}, n\right)=\frac{\bigoplus_{i=1}^{n}\left(1+T\left(\alpha_{i}\right)\right) \alpha_{i}}{\sum_{i=1}^{n}\left(1+T\left(\alpha_{i}\right)\right)}, \\
& T\left(\alpha_{i}\right)=\sum_{\substack{j=1 \\
j \neq i}}^{n} \operatorname{Sup}\left(\alpha_{i}, \alpha_{j}\right)
\end{aligned}
$$

and $\operatorname{Sup}\left(a_{i}, a_{j}\right)$ is the support for $a_{i}$ from $a_{j}$. The support satisfies the following three properties: 


$$
\begin{aligned}
& \text { 1) } \operatorname{Sup}\left(\alpha_{i}, \alpha_{j}\right) \in[0,1] \\
& \text { 2) } \operatorname{Sup}\left(\alpha_{i}, \alpha_{j}\right)=\operatorname{Sup}\left(\alpha_{j}, \alpha_{i}\right) \text {; } \\
& \text { 3) } \operatorname{Sup}\left(\alpha_{i}, \alpha_{j}\right) \geq \operatorname{Sup}\left(\alpha_{s}, \alpha_{t}\right) \text { if }\left|a_{i}-a_{j}\right|<\left|a_{s}-a_{t}\right| .
\end{aligned}
$$

Clearly, the HFPA and HFPG operator are two nonlinear weighted aggregation tools, and the weight $w_{i}=\frac{1+T\left(\alpha_{i}\right)}{\sum_{i=1}^{n}\left(1+T\left(\alpha_{i}\right)\right)}$ of argument $\alpha_{i}$ depends on all of the input arguments $\alpha_{j}(j=1,2, \mathrm{~K}, n)$ and allows the argument values to support each other in the aggregation process.

Theorem 1 Let $a_{i}=\left\{h_{a_{i}}, g_{a_{i}}\right\}(i=1,2, \mathrm{~K}, n)$ be a collection of DHFEs. The aggregated value using the DHFPA or DHFPG operator is also a DHFE, then

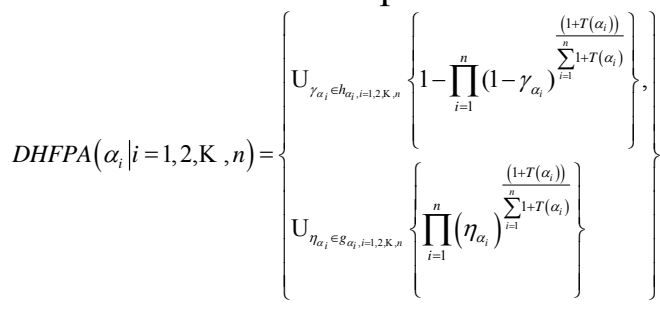

\section{Approach to multiple attribute group decision making with dual hesitant fuzzy information}

In the following, we utilize the proposed dual hesitant fuzzy power aggregation operator to develop some approaches to multiple attribute group decision making with dual hesitant fuzzy information:

Step 1. Transform the dual hesitant fuzzy decision matrix $A^{(t)}$ into the normalized dual hesitant fuzzy decision matrix $B^{(t)}=\left(\beta^{(t)}\right)_{m \times n}$ where:

$$
\beta_{i j}^{(t)}=\left\{\begin{array}{l}
\alpha_{i j}^{(t)}, \quad \text { for benefit attribute } \mathrm{g}_{i} \\
\left(\alpha_{i j}^{(t)}\right)^{c}, \text { for cost attribute } \mathrm{g}_{i}
\end{array}, i=1,2, \mathrm{~K}, m, j=1,2, \mathrm{~K}, n .\right.
$$

Step 2. Calculate the supports

$$
\operatorname{Sup}\left(\beta_{i j}^{(t)}, \beta_{i j}^{(p)}\right)=1-d\left(\beta_{i j}^{(t)}, \beta_{i j}^{(p)}\right) . t, p=1,2, \mathrm{~K} s, i=1,2, \mathrm{~K} m, j=1,2, \mathrm{~K} n
$$

which satisfy support conditions

Step 3.Utilize our operator to obtain the hesitant fuzzy elements for the alternatives

$$
\begin{aligned}
\beta_{i j} & =\operatorname{DHPA}\left(\beta_{i j}^{(1)}, \beta_{i j}^{(2)}, \mathrm{K}, \beta_{i j}^{(s)}\right) \\
& =\left(\frac{\bigoplus_{t=1}^{s} \omega_{t}\left(1+T\left(\beta_{i j}^{(t)}\right)\right)\left(\beta_{i j}^{(t)}\right)^{\lambda}}{\sum_{t=1}^{s} \omega_{t}\left(1+T\left(\beta_{i j}^{(t)}\right)\right)}\right)^{1 / \lambda}
\end{aligned}
$$

to aggregate all of the individual dual hesitant fuzzy decision matrices $B^{(t)}=\left(\beta_{i j}^{(t)}\right)_{m \times n}(t=1,2, \mathrm{~K}, s)$ into the collective dual hesitant fuzzy decision matrix $B=\left(\beta_{i j}\right)_{m \times n}$.

Step 4. Calculate the supports

$\operatorname{Sup}\left(\beta_{i j}, \beta_{i q}\right)=1-d\left(\beta_{i j}, \beta_{i q}\right) . i=1,2, \mathrm{~K} m, j, q=1,2, \mathrm{~K} n$

Step 5. Utilize the weights $w_{j}$ of attributes $g_{j}(j=1,2, \mathrm{~K}, n)$ to calculate the weighted support $T\left(\beta_{i j}\right)$ of DHFE $\beta_{i j}$ by the other DHFEs $\beta_{i q}(q=1,2, \mathrm{~K}, m$, and $q \neq j)$ 


$$
T\left(\beta_{i j}\right)=\sum_{\substack{q=1 \\ q \neq j}}^{n} \omega_{q} \operatorname{Sup}\left(\beta_{i j}, \beta_{i q}\right), i=1,2, \mathrm{~K} m, j, q=1,2, \mathrm{~K} n
$$

Step 6. Utilize the DHFPA operator to obtain the hesitant fuzzy elements for the alternatives

$$
\begin{aligned}
\beta_{i} & =\operatorname{DHPA}\left(\beta_{i 1}, \beta_{i 2}, \mathrm{~K}, \beta_{i n}\right) \\
& =\left(\frac{\bigoplus_{j=1}^{n} w_{j}\left(1+T\left(\beta_{i j}\right)\right)\left(\beta_{i j}\right)^{\lambda}}{\sum_{j=1}^{n} w_{j}\left(1+T\left(\beta_{i j}\right)\right)}\right)^{1 / \lambda}
\end{aligned}
$$

to aggregate all of the preference values $\beta_{i j}(j=1,2, \mathrm{~K}, n)$ in the ith line of $B$, and then derive the collective overall preference value $\beta_{i}$ of alternative $x_{i}(i=1,2, \mathrm{~K}, m)$.

Step 7. Rank the $\beta_{i}(i=1,2, \mathrm{~K}, m)$ in descending order.

Step 8. Rank all of the alternatives $x_{i}(i=1,2, \mathrm{~K}, m)$ and then select the best alternative in accordance with the collective overall preference values $\beta_{i}(i=1,2, \mathrm{~K}, m)$.

Now we consider one example. Energy is an indispensable factor for the socio-economic development of societies. Thus the correct energy policy affects economic development and environment, and so, the most appropriate energy policy selection is very important. Suppose that there are five alternatives (energy projects) $Y_{i}(i=1,2,3,4,5)$ to be invested, and four attributes $G_{j}(j=1,2,3,4)$ to be considered: $G_{1}$ : technological; $G_{2}$ : environmental; $G_{3}$ : socio-political; $G_{4}$ : economic. The attribute weight vector is $w=(0.15,0.3,0.2,0.35)^{T}$. Several decision makers are invited to evaluate the performance of the five alternatives. Utilizing DHFSs can take much more information into account, the more values we obtain from the decision makers, the greater epistemic certainty we have. So, We use DHHS to deal with such cases: $\left(g_{i j}\right)$ indicates the degree that the alternative $Y_{i}$ satisfies the attributes $G_{j}$ and $\left(h_{i j}\right)$ indicates the degree that the alternative $Y_{i}$ does not satisfy the attributes $G_{j}$. For an alternative under an attribute, although all of the decision makers provide their evaluated values, some of these values may be repeated. However, a value repeated more times does not indicate that it has more importance than other values repeated less times. For example, the value repeated one time may be provided by a decision maker who is an expert at this area, and the value repeated twice may be provided by two decision makers who are not familiar with this area. In such cases, the value repeated one time may be more important than the one repeated twice. To get a more reasonable result, it is better that the decision makers give their evaluations anonymously. We only collect all of the possible values for an alternative under an attribute, and each value provided only means that it is a possible value, but its importance is unknown. Thus the times that the values repeated are unimportant, and it is reasonable to allow these values repeated many times appear only once. The DHFS is just a tool to deal with such cases, and all possible evaluations for an alternative under the attributes can be considered as a DHFS. The results evaluated by the decision makers are contained in a dual hesitant fuzzy decision matrix, shown in Table 1. 
Table 1 dual hesitant fuzzy decision matrix.

\begin{tabular}{ccccc}
\hline & $G_{1}$ & $G_{2}$ & $G_{3}$ & $G_{4}$ \\
\hline$Y_{1}$ & $\{(0.5,04,0.3) ;(0.4,0.2)\}$ & $\{(0.9,0.8,0.7,0.1) ;(0.1,0)\}$ & $\{(0.5,04,0.2) ;(0.5,0.3,0.1)\}$ & $\{(0.9,0.6,0.5,0.3) ;(0.1)\}$ \\
$Y_{2}$ & $\{(0.5,0.3) ;(0.5,0.4,0.1)\}$ & $\{(0.9,0.7,0.6,0.5,0.2) ;(0.1)\}$ & $\{(0.8,0.6,0.5,0.1) ;(0.2,0.1)\}$ & $\{(0.7,0.4,0.3) ;(0.2,0)\}$ \\
$Y_{3}$ & $\{(0.7,0.6) ;(0.2)\}$ & $\{(0.9,0.6) ;(0)\}$ & $\{(0.7,0.5,0.3) ;(0.3,0.2)\}$ & $\{(0.6,0.4) ;(0.3,0.1)\}$ \\
$Y_{4}$ & $\{(0.8,0.7,0.4,0.3) ;(0.2,0.1)\}$ & $\{(0.7,0.4,0.2) ;(0.3,0.2)\}$ & $\{(0.8,0.1) ;(0.1)\}$ & $\{(0.9,0.8,0.6) ;(0.1,0)\}$ \\
$Y_{5}$ & $\{(0.9,0.7,0.6,0.3,0.1) ;(0.1)\}$ & $\{(0.8,0.7,0.6,0.4) ;(0.2,0)\}$ & $\{(0.9,0.8,0.7) ;(0)\}$ & $\{(0.9,0.7,0.6,0.3) ;(0.1)\}$ \\
\hline
\end{tabular}

Assume that the weights of the decision makers and attributes are known. We use Approach 1 to choose the best one:

Table2 Score values obtained by DHFPA pertor based on the dual hesitant Hamming distance and the ranking of alternatives

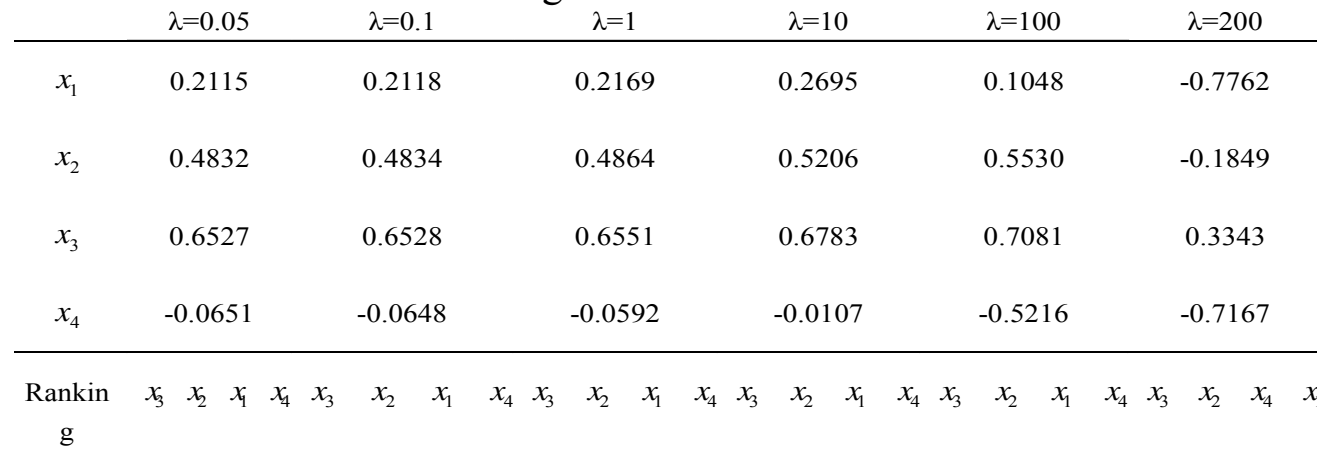

\section{Conclusion}

In this paper, we have developed some new operational laws for DHFEs.We have presented several new dual hesitant fuzzy aggregation operator called DHFPA. Moreover, we studied their properties. We have applied the proposed operator to develop one approache to multiple attribute group decision making in dual hesitant fuzzy environments. The prominent characteristic of our methods is that they can consider all the decision arguments and their relationships. Finally, by one typical real world example, we have illustrated the dual hesitant fuzzy multiple attribute group decision making process.

\section{Acknowledgements}

The authors acknowledge and extend their heartfelt gratitude to the Associate editor and anonymous reviewers for their insightful and constructive comments and suggestions that have led to an improved version of this paper. The corresponding author of paper is the Second author.

\section{References}

[1] B. Zhu, Z.S. Xu, M.M. Xia, Dual hesitant fuzzy sets, Journal of Applied Mathematics. 2012(2012), Article ID 879629, 13 pages. http://www.hindawi.com/journals/jam/2012/879629/.

[2] L. A. Zadeh, Fuzzy Sets, Information and Control 8 (1965) 338-353.

[3] K. Atanassov, Intuitionistic fuzzy sets, Fuzzy Set Syst. 20 (1986) 87-96.

[4] K. Atanassov, Intuitionistic Fuzzy Sets: Theory and Applications, Physica-Verlag, Heidelberg, 1999.

[5] V. Torra, Hesitant fuzzy sets, International Journal of Intelligent Systems 25 (2010) 529-539.

[6] V. Torra, Y. Narukawa, On hesitant fuzzy sets and decision, in: The 18th IEEE International Conference on Fuzzy Systems, Jeju Island, Korea, (2009)1378-1382.

[7] L.G. Zhou, H.Y. Chen, J.P. Liu. Generalized power aggregation operators and their applications 
in group decision making, Computers \& Industrial Engineering. 62 (2012) 989-999.

[8] Yager, R. R.. The power average operator. IEEE Transactions on Systems, Man and Cybernetics A, 31(2001), 724-731.

[9] Z.S. Xu., Yager, R. R. Power-geometric operators and their use in group decision making. IEEE Transactions on Fuzzy Systems, 18(2010), 94-105.

[10]L. Zhou, H. Chen, A generalization of the power aggregation operators for linguistic environment and its application in group decision making, Knowledge Based Syst. 26 (2012) 216-224.

[11]Z.S. Xu, Approaches to multiple attribute group decision making based on intuitionistic fuzzy power aggregation operators, Knowledge-Based Systems.24 (2011) 749-760.

[12]L.G. Zhou, H.Y. Chen, J.P. Liu, Generalized power aggregation operators and their applications in group decision making, Computers \& Industrial Engineering 62 (2012) 989-999.

[13]Z.M. Zhang, Generalized Atanassov's intuitionistic fuzzy power geometric operators and their application to multiple attribute group decision making, Information Fusion, 33(2013) 78-104. 